Revue d'histoire de l'Amérique française

REVUE D.HISTOIRE DE L'AMÉRIQUE FRANÇAISE

\title{
Du golfe Saint-Laurent aux côtes de Bretagne et de Normandie
}

(1713-1760)

\author{
L'Atlantique, un monde d'interactions et de solidarités
}

\section{Mario Mimeault}

Volume 67, numéro 1, été 2013

URI : https://id.erudit.org/iderudit/1026614ar

DOI : https://doi.org/10.7202/1026614ar

Aller au sommaire du numéro

Éditeur(s)

Institut d'histoire de l’Amérique française

ISSN

0035-2357 (imprimé)

1492-1383 (numérique)

Découvrir la revue

Citer cet article

Mimeault, M. (2013). Du golfe Saint-Laurent aux côtes de Bretagne et de Normandie (1713-1760) : l'Atlantique, un monde d'interactions et de solidarités. Revue d'histoire de l'Amérique française, 67(1), 5-31. https://doi.org/10.7202/1026614ar

\section{Résumé de l'article}

Cet article entend démontrer les liens qui cimentaient la communauté maritime française en considérant les relations déployées à l'échelle des individus entre les littoraux de la France et de la Nouvelle-France. Nous posons l'hypothèse que l'univers atlantique, plus qu'un espace de mouvements, a été constitué d'un faisceau de relations interpersonnelles développées entre les points de départ des travailleurs de la mer, ceux qu'on appelle les pêcheurs, et leurs lieux d'implantation en Amérique. L'ensemble de ces rapports constitue une facette immatérielle mais bien réelle de la réalité halieutique, celle de l’interdépendance et des solidarités maintenues malgré les distances. 


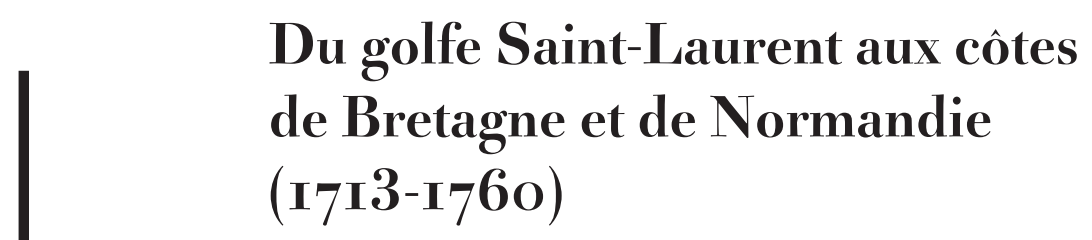

L'Atlantique, un monde d'interactions et de solidarités ${ }^{\mathrm{I}}$

Mario Mimeault

Chercheur indépendant

RÉSUMÉ • Cet article entend démontrer les liens qui cimentaient la communauté maritime française en considérant les relations déployées à l'échelle des individus entre les littoraux de la France et de la Nouvelle-France. Nous posons l'hypothèse que l'univers atlantique, plus qu'un espace de mouvements, a été constitué d'un faisceau de relations interpersonnelles développées entre les points de départ des travailleurs de la mer, ceux qu'on appelle les pêcheurs, et leurs lieux d'implantation en Amérique. L'ensemble de ces rapports constitue une facette immatérielle mais bien réelle de la réalité halieutique, celle de l'interdépendance et des solidarités maintenues malgré les distances.

ABSTRACT - This paper illustrates the ties that were binding the French marine community by examining the ongoing relations between the residents of the costal regions of France and New France. The article is based on the hypothesis that the Atlantic Ocean was more than a passage, that it was in fact a nexus of interpersonal relations developed between the starting points of the sea workers - the fishermen- and their new homes in America. These relations constitute a facet which, although immaterial, is a concrete manifestation of a fishery reality, that of interdependence and solidarity maintained regardless of distances.

D es rencontres tenues tant au Canada qu'en France ces dernières années ont eu comme thème l'analyse de sous-espaces régionaux

1. L'auteur tient à remercier les lecteurs anonymes de la RHAF. Leurs commentaires et suggestions ont permis de corriger et d'enrichir l'article. Il exprime aussi sa gratitude à Yolaine Sirois pour la correction de son orthographe. 
en regard du cadre atlantique français ${ }^{2}$. Sensibilisé à cette perspective, nous sommes intervenu à deux reprises à propos de la dynamique qui animait les relations intercontinentales. Une première fois à La Rochelle pour souligner l'héritage que les pêcheurs et entrepreneurs métropolitains ont partagé avec les gens de la Nouvelle-France à la faveur d'une cofréquentation des aires de transformation de la morue. Nous marquions par là le passage d'une rive à l'autre de l'océan non seulement des hommes, mais aussi des connaissances régissant leurs activités. Une seconde fois à Shippagan, pour aborder l'interaction qui pouvait avoir existé entre l'Ancien et le Nouveau Monde sous l'angle du savoir transmis au-delà des distances puis renouvelé et, finalement, adapté à de nouveaux besoins. L'apport de ces connaissances à notre patrimoine collectif suggérait rien de moins que l'existence d'une communauté inter-rive bien vivante ${ }^{3}$.

Cette orientation de la recherche s'inscrit dans la continuité des études des dernières décennies. Dans les années 1960, Charles de La Morandière a publié une histoire de la pêche française de la morue en Atlantique Nord que l'on peut considérer comme le point d'appui d'un nouvel élan. Le traitement de son sujet relevait de l'observation davantage que de l'analyse, mais il suffisait à éveiller l'intérêt des chercheurs. Par la suite, les historiens ont contribué dans la foulée de la Nouvelle histoire sociale à l'enrichissement des thèmes les plus divers suivant des approches variées: les pêches maritimes, la chasse à la baleine, les grands ports de mer, le commerce atlantique, les gens de mer ${ }^{4}$. Après les hommes, leurs activités

2. Colloque international, Brouage/La Rochelle, 27-28 septembre 2007 : Entre Golfe du Saint-Laurent et Centre-Ouest français : contacts, échanges et développements comparés des littoraux, GERHICO-IEAQ, Université de Poitiers, Université de La Rochelle et Université de Moncton. ACFAS, Congrès national des sociétés historiques et scientifiques, 133 congrès, Québec, 2008: Migrations, transferts et échanges de part et d'autre de l'Atlantique. Colloque international, Shippagan, 11 au 14 août 2009: Développement comparé des littoraux du Golfe Saint-Laurent et du Centre-Ouest français : d'hier à Aujourd'hui, Université de Moncton, Campus de Shippagan.

3. Mario Mimeault, «L'industrie canadienne de la pêche à la morue (1663-1758) - Naissance d'une industrie au savoir emprunté », dans Michael Augeron, Jacques Péret et Thierry Sauzeau, dir., Le golfe du Saint-Laurent et le Centre-Ouest français - Histoire d'une relation singulière (XVII ${ }^{e}$-XIX ${ }^{e}$ siècles) (Rennes, Presses Universitaires de Rennes, 2010), 17-36. Mario Mimeault, «Exemples d'un savoir transmis, renouvelé puis adapté dans l'industrie de la pêche dans le golfe Saint-Laurent ", dans Nicolas Landry, Jacques Péret et Thierry Sauzeau, dir., Développement comparé des littoraux du golfe Saint-Laurent et du Centre-Ouest français d'hier à aujourd'hui (Moncton, Université de Moncton, 2012), 141-162.

4. À tous ceux-là nous pourrions ajouter la navigation atlantique, la navigation à voile, la course en mer, la piraterie, le pilotage, les phares et leurs gardiens, l'outillage du pêcheur et bien d'autres thèmes. Voici quelques ouvrages à titre indicatif: Charles de La Morandière, Histoire de la pêche française de la morue dans l'Amérique septentrionale (Paris, G.-P. Maisonneuve et Larose, 1962), 1023 p. Alain Cabantous, La mer et les hommes - Pêcheurs et matelots dunkerquois de Louis XIV à la Révolution (Dunkerque, WesthoekÉdition, 1980), 351 p. Jacques Mathieu, Le commerce entre la Nouvelle France et les Antilles au XVIII ${ }^{e}$ siècle (Montréal, Fides, 1981), 276 p. Gilles Proulx, Entre France et Nouvelle-France (La Prairie, Parcs Canada/ 
et leurs points d'ancrage, le courant de recherche le plus récent oriente ses efforts sur leur terrain d'occupation, l'océan Atlantique. Selon les tenants de cette approche, issue du monde anglo-saxon, l'Atlantique "aurait constitué un "pont" favorisant un processus d'interaction et de convergence qui a modifié à jamais les perceptions, les identités, les économies sur chacun des continents [Europe, Amérique, Afrique] $]^{5} »$. Cette nouvelle vision de l'univers inter-rive a animé en France des groupes de recherche qui se sont penchés en particulier sur les liens développés entre les littoraux du golfe Saint-Laurent et ceux du Centre-Ouest de l'Hexagone ${ }^{6}$. C'est en continuité avec la réflexion de ces cercles de chercheurs que nous nous y sommes aussi intéressé.

En effet, un vent de renouveau se fait sentir dans le champ des migrations, des guerres France-Angleterre ou des rapports commerciaux, et plus particulièrement pour la circulation des bateaux, des hommes et des idées ${ }^{7}$. Pour sa part, l'étude des communications prévalant dans le monde

Éditions Marcel Broquet, 1984), 197 p. Michel Mollat, Histoire des pêches maritimes en France (Paris, Bibliothèque historique Privat, 1987), 407 p. Jean-François Brière, La pêche française en Amérique du Nord au XVIII siècle (Montréal, Fides, 1990), 270 p. Henri Veyrier, Cent ans de pêche à Terre-Neuve (Paris, Kronos, 1991), 3 vol. Jean-Pierre Proulx, Les Basques et la pêche de la baleine au Labrador au XVIe siècle (Ottawa, Environnement Canada, 1992), 118 p. Peter Pope, Fish into Wine: The Newfoundland Plantation in the Seventeenth Century (Chapel Hill, University of North Carolina Press, 2004), xxvi-463 p. Nicolas Landry, Plaisance (Terre-Neuve) 1650-1713 - Une colonie française en Amérique (Québec, Septentrion, 2004), 406 p. Nicolas Landry, Éléments d'histoire des pêches. La péninsule acadienne du Nouveau-Brunswick, 1890-1950 (Québec, Septentrion 2005), 311 p. Mario Mimeault, Destins de pêcheurs: Les Basques en Nouvelle-France (Québec, Septentrion, 2011), 203 p.

5. Silvia Marzagalli, «L'histoire atlantique en Europe», Nuevo Mundo/Mundos Nuevos [Mis en ligne le 24 septembre 2008], paragraphe 1: http://nuevomundo.revues.org/42463 (Consulté le 28 mai 2014). L'histoire atlantique est encore en balbutiement. Lire sur le concept: Bernard Bailyn, Atlantic History: Concept and Contours (Cambridge, Harvard University Press, 2005), 111 p. Jack P. Greene et Philip D. Morgan, dir., Atlantic History: A Critical Appraisal (Oxford, Oxford University Press, 2009), x-371 p. Au Québec, le lecteur peut en référer au travail du Groupe d'histoire de l'Atlantique français du département d'histoire de l'Université McGill : http:/ / atlantique.mcgill.ca/content.php?db=home (Consulté le 28 mai 2014).

6. Plusieurs mémoires de maîtrise sont issus de cet intérêt pour l'Amérique: Laura Huet, «L'économie du naufrage en Nouvelle-France entre 1715 et 1763 », Mémoire de Master I (histoire), Université de Poitiers, 2012. Olivier Puaud, «Les marins d'Amérique du Nord rapatriés dans les ports du littoral français. Exemple dans les ports de Rochefort et de La Rochelle 1770-1816», Mémoire de Master II (histoire), Université de Poitiers, 2005-2006. Fabrice Lanvin, «La Rochelle et la Grande Pêche en Amérique du Nord au XVIII siècle (1713-1789)", Mémoire de Master 2 (histoire), Université de La Rochelle, 2010. Cédric Brothaire, «Les gens de mer de l'île Royale: vie et mœurs (1713-1758)», Mémoire de Master I (histoire), Université de La Rochelle, 2001.

7. Jean-Paul Hervieu, Gilles Désiré dit Gosset et Éric Barré, dir., Les Normands et l'outre-mer (Caen, Annales de Normandie 2011), 414 p. Leslie Choquette, De Français à paysans (Québec/Paris, Septentrion/ Presses de l'Université de Paris-Sorbonne, 2001), 323 p. Robert Larin, Brève histoire du peuplement en Nouvelle-France (Québec, Septentrion, 2000), 229 p. Gaston Deschênes et Denis Vaugeois, Vivre la Conquête, 2 vol. (Québec, Septentrion, 2013/2014), 250 p., 350 p. Kenneth J. Banks, Chasing Empire - Across the Sea - Communications and the State in the French Atlantic, 1713-1763 (Montréal, McGill-Queen's University Press, 2003), 319 p. Jonathan R. Dull, The French Navy and the Seven Years's War (Lincoln \& Londres, University of Nebraska Press, 2005), 445 p. Nicolas Landry, Jacques Péret et Thierry Sauzeau, Développement comparé 
halieutique demeure encore un champ en développement au Canada français. Ce texte entend démontrer les liens qui cimentaient la communauté maritime française en considérant les relations déployées à l'échelle des individus entre les littoraux de la France et de la Nouvelle-France. Nous posons l'hypothèse que l'univers atlantique, plus qu'un espace de mouvements, a été constitué d'un faisceau de relations interpersonnelles développées entre les points de départ des travailleurs de la mer, ceux qu'on appelle les marins et les pêcheurs, et leurs lieux d'implantation en Amérique. L'ensemble de ces rapports constitue une facette immatérielle mais bien réelle de la réalité halieutique, celle de l’interdépendance et des solidarités maintenues malgré les distances.

Nous abordons ce sujet en nous référant à l'expérience des gens impliqués dans une facette ou l'autre de la vie en mer. En utilisant des tranches de vie à la manière de Dick Hoerder, démarche que l'on a baptisée The life course approach, ou Histoire de vie, nous verrons que ce sont les individus qui ont donné un sens à l'univers atlantique. Nous rappelons d'abord l'importance du trafic des morutiers aux fins de poser de manière concrète les bases de nos observations. Puis nous y superposons une dynamique de relations constantes entre la France et la Nouvelle-France. Nous sommes bien conscient que cette position contraste avec la croyance suivant laquelle les métropolitains impliqués dans les pêches ou la vie en mer, et dont certains deviennent résidants du territoire, ont été complètement isolés du monde et de leurs familles en France. Tout au contraire de ce courant de pensée, nous nous intéresserons à ce qui a constitué les éléments d'un maillage transocéanique à l'échelle des individus, les liens d'affaires ou professionnels, de provenance, d'amitié ou de famille.

Notre attention se porte principalement sur deux sous-espaces régionaux habités ou fréquentés par les pêcheurs, la partie québécoise du golfe Saint-Laurent, du côté nord-américain, et une partie du nord-ouest français, soit les côtes de la Bretagne et de la Normandie chez qui, comme l'ont observé nombre d'auteurs, les relations avec l'Amérique sont parmi les plus régulières.

Cette approche du sujet est susceptible d'apporter une nouvelle contribution à l'histoire atlantique autant qu'à celle des migrations. En nous arrêtant à des régions reliées de part et d'autre de l'océan, tout en nous

des littoraux du golfe Saint-Laurent et du Centre-Ouest français d'hier à aujourd'hui (Moncton, Université de Moncton, 2012), 325 p. Michael Augeron, Jacques Péret et Thierry Sauzeau, Le golfe du Saint-Laurent et le Centre-Ouest français - Histoire d'une relation singulière (XVII ${ }^{e}$-XIX ${ }^{e}$ siècles) (Rennes, Presses Universitaires de Rennes, 2010), 363 p. 
fixant sur le maintien des relations interpersonnelles des gens qui se partagent entre les deux mondes, nous croyons renforcer l'image d'un univers qui se définit par son unité. À cette fin, tout un éventail d'études et de documents dispersés a été rassemblé. Les travaux des historiens qui nous ont précédé ont apporté des informations et des données qui ont permis de poser le cadre de notre recherche. Pour le reste, les Archives des Colonies en France, Bibliothèque et Archives Canada à Ottawa ainsi que Bibliothèque et Archives nationales du Québec conservent l'essentiel du corpus documentaire auquel nous avons eu recours: correspondance officielle, actes notariés, témoignages de liberté au mariage, fonds familiaux, registres d'état civil, etc. À cela s'ajoutent certains sites Internet qui diffusent, au profit des chercheurs, des listes de passagers et rôles d'équipage porteurs d'informations nouvelles sur les émigrants qui ont franchi l'Atlantique. Cet ensemble archivistique ouvre la porte sur le golfe SaintLaurent en tant que champ d'exploitation métropolitain.

\section{LE GOLFE SAINT-LAURENT, UN MONDE DE MOUVEMENT}

John A. Dickinson souligne le fait que l'Amérique est un territoire de mouvement, mais, pourrions-nous ajouter, l'océan Atlantique constitue un espace tout aussi bouillonnant ${ }^{8}$. Les faits parlent d'eux-mêmes. Quand on y regarde de près, le milieu intercontinental est pendant toute la période coloniale française le théâtre d'un véritable chassé-croisé de navires conduisant les métropolitains en Amérique et les Canadiens à la mère patrie 9 .

C'est ainsi que l'on peut se remettre en mémoire le brassage des populations qui ont sillonné l'Atlantique à partir des côtes françaises entre 1492 et 1760 et qui ont abouti ou passé par le golfe Saint-Laurent. Nous pensons à la traversée des colons et des filles du roy dans les années 1660 et 1670, à l'envoi de renforts militaires à tout moment $(1690,1758,1760)$, à la déportation des colons anglais de Terre-Neuve par d'Iberville en 1697, aux déplacements des colons-pêcheurs de Plaisance vers l'île Royale (aujourd'hui île du Cap Breton, N.-É.) en 1713, à la déportation de 400 habitants de Louisbourg vers Boston en 1745, à celui des Acadiens vers les côtes de la Nouvelle-Angleterre en 1755, puis des Gaspésiens vers la Bretagne en 1758 et, enfin, au renvoi des officiers civils et de la garnison française en 1760. De toute évidence, plus qu'un monde en mouvement,

8. John A. Dickinson, «Préface», dans Robert Larin, Canadiens en Guyane (Québec/Paris, Septentrion/ PUPS, 2006), 15-16.

9. R. Larin, op. cit., 26-62. 
l'espace maritime joignant l'ancien et le nouveau continent doit être perçu non pas comme une césure, mais davantage comme un trait d'union entre la colonie et la mère patrie ${ }^{10}$.

Le déferlement annuel des pêcheurs bretons et normands de ce côté-ci de l'Atlantique nous en offre un exemple probant. Un trafic intense s'observe entre l'extrémité de la péninsule gaspésienne et les ports français. Quelques personnes en ont témoigné. En 1672, Nicolas Denys affirme qu'il se trouve onze navires par année dans l'anse de Percé. En 1686, quand l'intendant de Meulles visite l'établissement local, ce dernier dit y rencontrer les capitaines de dix morutiers. Pour juger de l'importance de cette présence métropolitaine sur les côtes, il faut savoir que le port de Québec, loin à l'intérieur du continent, ne bénéficiera d'un taux de fréquentation équivalent que dans les décennies 1713 à 1743 . Et cette estimation ne tient pas compte du nombre de bateaux de pêche qui jetaient leurs lignes dans la baie de Gaspé, au Labrador, à Terre-Neuve et en Acadie, où le port de Louisbourg recevait à lui seul de 130 à 150 navires de commerce et jusqu'à 400 navires pêcheurs chaque anné $e^{11}$.

En fait, de 1720 à 1758, les ports de Granville et de Saint-Malo laissent partir chaque printemps des dizaines de morutiers en direction du golfe Saint-Laurent. En quatre décennies, de 1720 à 1758, les armateurs de ces deux grands centres maritimes appareillent 2731 bateaux de pêche ${ }^{12}$. L'enregistrement de ces vaisseaux montre que leurs équipages occupent tous les points du golfe. Alors que 1559 navires gagnent le Grand Banc, 1155 jettent l'ancre sur les côtes de Terre-Neuve (au Petit Nord), 636 à la Grand

10. À nos yeux, la mer ne saurait être une barrière en aucune manière. Soulignant la présence des plans d'eau qui entourent la Gaspésie (le fleuve Saint-Laurent, le golfe du même nom, la baie des Chaleurs et l'Atlantique), nous écrivions que "dans tous les cas..., d'où que nous pouvions porter le regard, ces étendues d'eau constituent le trait d'union avec l'extérieur ", Mario Mimeault, La Gaspésie. Histoire en bref (Québec, IQRC, 2004), 8.

11. Archives nationales d'outre-mer (ANOM), Jacques de Meulles, «Règlement fait par M. de Meulles à l'île percée», COL C11D 2/fol.8-9 le 19 juin 1686: http://bd.archivescanadafrance.org/sdx-222-acfpleade- $2 /$ acf $/$ search-acf.xsp?sq1 $=\mathrm{r} \% \mathrm{C} 3 \% \mathrm{~A} 9$ glement + perc $\% \mathrm{C} 3 \% \mathrm{~A} 9 \&$ fi1 $1=$ fulltext $\&$ sq $2=\&$ fi2 $2=$ fulltext \&sq3 $=\& f i 3=$ fulltext $\&$ ed $=1686 \& \mathrm{dbeg}=\&$ dend $=\&$ frepo $=\&$ forig $=\& \mathrm{x}=0 \& \mathrm{y}=0 \#$ resume $($ Consulté le 2 octobre 2013). Nicolas Denys, «Description géographique et historique des costes de l'Amerique septentrionale avec l'Histoire naturelle du Pais", dans Clarence-Joseph d'Entremont, Nicolas Denys, Sa vie et son ceuvre (Yarmouth, N.-É.), Imprimerie Lescarbot, 1982), 129 colonne 228, 130 colonne 232. Pour plus d'informations sur le trafic maritime qui a cours dans les eaux du golfe Saint-Laurent, lire K. J. Banks, op. cit., 73-76; John Willis, «The Scale of Postal Communication in New France in context », dans Muriel Le Roux, dir., Postes d'Europe, XVIII siècle - XXI' siècle, jalons d'une histoire comparée (Paris, Comité pour l'histoire de La Poste, 2007), 127-148. Ce dernier texte est repris en français sous le titre: «Les échelles de la communication postale en Nouvelle-France», dans Yves Frenette, Marcel Martel et John Willis, dir., Envoyer et recevoir. Lettres et correspondances dans les diasporas francophones (Québec, PUL, 2006), 127-144.

12. Nos statistiques se fondent sur celles avancées par Jean-François Brière, La pêche française en Amérique du Nord au XVIII' siècle (Montréal, Fides, 1990), 152-160. 
Bay (Labrador), 615 à l'île Royale et 386 sur les côtes de Gaspé (lire Gaspésie).

Quant aux travailleurs de la mer qui accompagnent cette armada, ils sont légions. Pour en juger, il faut en référer au peuplement de la Nouvelle-France. Les historiens s'accordent pour dire qu'environ 30000 émigrants français ont traversé dans la colonie (7000 de plus si on prend l'Acadie en compte) depuis ses débuts jusqu'à sa cession à l'Angleterre en 1763. Cette donnée comprend autant les gens qui se sont implantés définitivement que les employés saisonniers ou temporaires engagés dans l'administration, la domesticité, le commerce, la traite des fourrures et dont la très grande majorité est retournée en France ${ }^{13}$.

Il semble toutefois que les études conduisant à cette évaluation fassent abstraction des hommes d'équipage, de ces marins et pêcheurs français qui ont gagné annuellement les côtes de la Nouvelle-France et que l'on peut classer au rang des travailleurs saisonniers. L'opération aurait eu pour effet de gonfler considérablement les données. L'armement des morutiers mobilisait, en effet, des centaines, voire des milliers d'hommes chaque année. Ainsi, en recentrant notre attention sur la côte de Gaspé et pour ne prendre en compte qu'une seule année, celle de 1725, nous voyons que neuf navires normands y ont côtoyé les équipages de treize morutiers malouins. Au total, vingt-deux navires pêcheurs se sont alors partagé les anses de la péninsule. Cela représente en termes d'effectifs humains 1142 travailleurs disséminés entre le cap des Rosiers et le cap d'Espoir $(125 \mathrm{~km})$. Cela paraît peu en regard des 30000 personnes recensées par les chercheurs, mais ce n'est qu’une donnée partielle. En étendant le regard à l'ensemble du golfe, on verrait cette année-là 3083 pêcheurs bretons et normands au travail et, en prenant du recul dans le temps, c'est

13. Il semble que ces données doivent être réévaluées. Leslie Choquette, dont les travaux font autorité, laisse entendre que l'on pourrait même doubler ce chiffre et parle de 67000 potentiels immigrants français. Et Choquette ne tient pas compte des travailleurs saisonniers, ce qui ne signifie pas qu'elle ignore leur existence: Leslie Choquette, De Français à paysans, op. cit., 4, 18-20, 52, 178-179. Les pêcheurs, non plus que les travailleurs saisonniers, ne sont pas davantage considérés par Jean-Pierre Poussou dans son étude sur la mobilité des Français. Ce dernier met plutôt l'accent sur la migration fondatrice du Canada. Jean-Pierre Poussou, "Mobilité et migration », dans Jacques Dupâquier, Histoire de la population française (Paris, PUF, 1988), vol. 2, 99-143. Ces mêmes données (30 000 immigrants pour la Nouvelle-France) semblent acceptées par Yves Landry, «Les immigrants en Nouvelle-France: bilan historiographique et perspective de recherche", dans Philippe Joutard et Thomas Wien, dir., Mémoires de Nouvelle-France-De France en Nouvelle-France (Rennes, PUR, 2005), 68, ainsi que Georges Coste et Dominique Guillemet, "Mémoires de la Nouvelle-France en Poitou-Charentes. De l'histoire à l'invention et invention du patrimoine», dans Philippe Joutard et Thomas Wien, dir., Mémoires de Nouvelle-France..., 305-318. Voir aussi Mario Boleda, "Trente mille Français à la conquête du Saint-Laurent », Histoire Sociale/Social History, 23, 45 (1990): 153-177. 
une marée humaine que l'on apercevrait. Considérant les pêcheurs qui ont mis le pied dans un établissement ou l'autre du golfe Saint-Laurent entre 1713 et 1758 , on retrouverait 177025 personnes à l'œuvre ${ }^{14}$.

\section{DES LIENS D'AFFAIRES INTER-RIVES}

Comme on le voit, les eaux du golfe Saint-Laurent, et à plus forte raison ses rives, sont des lieux d'intenses fréquentations. Les dizaines de morutiers qui traversent l'Atlantique chaque année déversent des milliers de travailleurs de la mer, mais jamais le lien qui les unit avec la mère patrie n'est rompu. Il y a ceux qui reviennent à bon port, bien sûr, mais il y a aussi ceux qui restent en Amérique. Tout ce monde garde le contact. Ainsi se créent des relations humaines aux facettes multiples, souvent reliées entre elles. Au premier titre, une catégorie de personnes alimentent le trafic transocéanique et en retirent tout le bénéfice possible pour leurs affaires: des entrepreneurs comme Pierre Revol, des marins au long cours comme Jean Barré, des investisseurs comme Charles Blondel, presque tous de Granville. Leur carrière incarne rien de moins que les relations intercontinentales dont nous faisons état ici.

Le premier, Pierre Revol, présente un parcours de vie qui illustre une facette bien particulière des liens intercontinentaux. Faux-saunier condamné à l'exil, la Nouvelle-France apparaît dans son cas comme une terre d'expiation pour faute commise en mère patrie, ce qui ne l'a pas empêché de mener une carrière dynamique. Ici même, une fois installé au Nouveau Monde, il exploite des installations de pêche et de transformation du poisson dans la baie de Gaspé entre les années 1752 et 1758. Ce faisant, il devient un occupant illégal du territoire en raison des restrictions imposées par l'Ordonnance de la Marine de 1681. Et pourtant les autorités, qui voyaient dans sa présence le moyen de contrôler un espace qu'elles ne pouvaient défendre autrement, n’étaient que trop heureuses de lui accorder leur bénédiction ${ }^{15}$. Or, ce dernier, aux prises avec

14. Ces chiffres sont appuyés sur les statistiques fournies par Jean-François Brière. Les recherches de Gervais Carpin l'ont amené à penser que ce contingent pourrait être encore plus nombreux, même au siècle précédent: «Sur un siècle, même si on garde à l'esprit que nombre de ces pêcheurs accomplissaient plusieurs campagnes au cours de leur vie, il n'est pas invraisemblable d'écrire que plusieurs dizaines (même centaines) de milliers de Picards, Normands, Bretons, Poitevins, Aunisiens, Saintongeais, Guyanais, Gascons et Basques avaient déjà séjourné en Nouvelle-France quand Champlain y vint»: Gervais Carpin, "Les migrations vers la Nouvelle-France au temps de Champlain», dans Raymonde Litalien et Denis Vaugeois, dir., Champlain. La naissance de l'Amérique française (Québec, Éditions Nouveau Monde/Septentrion, 2004), 177.

15. Mario Mimeault, «Pierre Revol», dans G. Deschênes et D.Vaugeois, Vivre la Conquête, op. cit., vol. 1, $151-161$. 
un manque criant de main-d'œuvre dans la colonie, s'est tourné vers la mère patrie pour recruter son personnel et demander ensuite la concession des lieux:

Suppliant humblement André Arnoux et Pierre Revol qui ont l'honneur de vous représenter très respectueusement qu'ayant commencé un établissement sur la côte de Gaspé depuis trois années et ayant déjà établi quarante habitants à qui ils ont fait défricher des terres ${ }^{16}$.

Les recherches pour retrouver ces engagés n'ont abouti que dernièrement. L'équipe du site Internet Migrations ayant relevé les rôles des équipages et des passagers qui ont gagné les rives du golfe Saint-Laurent depuis Granville et Saint-Malo, il est apparu que 148 passagers se sont embarqués depuis ces ports pour Gaspé entre 1753 et 1755. C'est beaucoup plus que le nombre avancé par Revol dans sa requête, mais plusieurs se sont éparpillés sur la côte de Gaspé ${ }^{17}$. Seulement pour le Mont-Louis, nous avons repéré six engagés sur la trentaine que Michel Mahiet entretenait à son poste de pêche ${ }^{18}$.

Le cas d'un autre ressortissant français, Jean Barré, sans être similaire, illustre parfaitement le lien d'affaires qui réunit les deux côtés de l'Atlantique. Originaire de Saint-Pair, mais résidant de Granville, comme toute sa famille, ce capitaine a connu une brillante et fructueuse carrière intercontinentale. D'abord pêcheur puis marin au long cours, il dirige dans les années 1730 plusieurs expéditions de pêche sur le Grand Banc et dans le golfe Saint-Laurent pour le compte de marchands et avitailleurs granvillais. Il se fait ensuite industriel de la pêche et s'installe à l'île Royale, où il investit dans un commerce transocéanique avec ses frères Thomas, demeuré à Granville, et Nicolas, qui reste sur l'île. Nicolas dirige les opé-

16. BAC, Archives des colonies - MG. 1, C C, vol. 8, p. 3-5, Lettre de Revol et Arnoux, le 18 juillet 1755 ; MG. 1, C A, vol. 99, p. 303-306 (bobine F-99). Voir: http:// collectionscanada.gc.ca (Consulté le 14 octobre 2013).

17. Pour la liste des passagers, nous renvoyons nos lecteurs à Migrations à la rubrique «Navires de nos ancêtres" : www.migrations.fr/Derniers\%20Passagers.htm (Consulté le 14 octobre 2013). Quant aux possibles contrats d'engagement des pêcheurs par Revol, il semble que tout espoir de les retrouver soit vain puisqu' “en juin 1944, les Alliés ont bombardé Saint-Lô et ses archives. » Courriel de Jocelyne NicolQuillivic à Mario Mimeault, le 17 octobre 2011. Pour qui voudrait élargir la recherche à l'ensemble des pêcheurs œuvrant dans le golfe Saint-Laurent, il n'est pas sans intérêt de souligner l'apport potentiel des inventaires après-décès des pêcheurs engagés à Plaisance et à l'île Royale, lesquels mentionnent souvent le lieu d'origine des engagés et parfois d'entrepreneurs en pêche. Les études de Nicolas Landry et de Berton Alexander Balcom fourniront à cet égard de multiples pistes: Nicolas Landry, Plaisance (TerreNeuve) 1650-1713 - Une colonie française en Amérique; B. A. Balcom, La pêche à la morue à l’Ile Royale l7l3-l758 (Ottawa, Parcs Canada, 1984), 94 p.

18. Mario Mimeault, "Michel Mahiet et la pêche côtière au Mont-Louis 1744-1759", Revue d'histoire de la Gaspésie, 17, 2 (avril-juin 1979): 4-19. 
rations de transformation du poisson sur le terrain, Jean s'occupe du transport de la production outre-Atlantique et Thomas veille à l'écoulement des stocks sur le marché en France. C'est l'exemple même d'une entreprise intégrée dont les rouages sont axés sur la mise en valeur des richesses de l'Atlantique en appui sur des structures qui ont leurs assises des deux côtés de l'océan. Conséquence, un va-et-vient de part et d'autre de la grande mare témoigne de la continuité des affaires familiales ${ }^{19}$.

Le profil de Charles Blondel ressemble un peu à celui des deux précédents personnages. Pêcheur, capitaine de navire, armateur, entrepreneur, il fera une carrière partagée entre sa province natale et la Nouvelle-France. Originaire de Tanu, près de Granville, on le trouve pour la première fois en 1722, à trente-cinq ans, pêcheur sur le Grand Banc. Le reste de la décennie est ponctué par de nombreux passages outre-mer dont quelques-uns sur la côte de la Gaspésie. Au début des années 1730, il engage des pêcheurs pour travailler à son compte à l'île Royale. Cette expérience est marquée par la perte d'une chaloupe et de son équipage, mais cet accident de travail ne le décourage pas. Peu de temps après, Blondel s'associe à François Aubut pour exploiter un établissement de pêche à Grande-Rivière, en Gaspésie. C'est l'occasion de se lancer dans des opérations d'envergure, d'autant qu'il s'était construit quelques années auparavant deux navires de pêche à Gaspé, L'Aventurier en 1736 et la Marie-Anne en $1740^{20}$.

Malgré son installation dans les eaux du golfe Saint-Laurent, ses liens avec sa province d'origine ne sont pas coupés. En témoigne le fait que ses deux bateaux construits en Amérique sont enregistrés à Granville, alors qu'ils auraient bien pu l'être à Québec, et que tous les membres de ses équipages sont de l'arrière-pays granvillais. À l'occasion, il partage ses frais d'armement avec des négociants de sa ville natale. Charles Blondel possédera par la suite plusieurs autres bateaux aussi montés par des gens de la Normandie, mais il conservera toujours ses bases opérationnelles dans la région de Grande-Rivière ${ }^{21}$.

19. Robert Larin et Mario Mimeault, avec la collaboration d'André Bardou, "Entre France et Nouvelle-France - Jean Barré (1694-1776), pêcheur, entrepreneur et capitaine au long cours ", La Revue de la Manche, 52, fascicule 208 (avril 2010): 31-47.

20. Mario Mimeault et Fabien Synnett, avec la collaboration de Ginette Roy, Gaspé au fil du temps (Gaspé, Ville de Gaspé, 2009), 65 s.

21. Voir pour ses traversées entre la France et les côtes de la Gaspésie ainsi que les rôles de ses équipages le site Migrations, op. cit. BAC, Conseil supérieur de Louisbourg (R 11577-38-8-F, anciennement MG1, G2, vol. 181), janvier 1732, p. 283 des transcriptions, Procédures touchant le décès de pêcheurs à l'emploi de Charles Blondel. 
Au final, il appert que les parcours de Revol, Barré et Blondel se ressemblent. Tous les trois ont mené une carrière à cheval entre deux continents. Les eaux du golfe Saint-Laurent furent pour eux une école d'apprentissage et un terrain professionnel, alors que l'Atlantique leur permettait de maintenir le lien avec une main-d'œuvre normande et un marché métropolitain.

\section{DES PÊCHEURS MOBILES MAIS BIEN PRÉSENTS}

Jusqu'ici, nous avons signalé l'intensité de trafic transocéanique mettant en cause les navires et les hommes. Nous avons mentionné l'existence de postes de pêche exploités conjointement par des entrepreneurs canadiens de première génération et des investisseurs métropolitains. Nous avons fait état de liens d'affaires qui s'étaient tissés de part et d'autre de l'Atlantique, mais qu'en est-il du personnel auxiliaire, des hommes à la base de leurs opérations, les pêcheurs?

Le déplacement de cette main-d'œuvre d'une rive à l'autre de l'Atlantique présente les caractéristiques d'une migration professionnelle de type transocéanique ${ }^{22}$. Si la mobilité des travailleurs est saisonnière, comme dans les campagnes où leurs allées et venues dépendent des périodes des moissons, des vendanges ou de la transhumance, la migration des pêcheurs a spécifiquement rapport aux activités en haute mer et à la mobilité de la morue. Ces départs pour la grande pêche s'accompagnent dans l'ensemble par un retour au port d'attache chaque automne, ces trajets à répétition pouvant s'effectuer pendant de nombreuses années pour un même homme.

Les allégations voulant que ce type de déplacement de population se soit effectué sans peuplement véritable et que, si implantation il y a eu, elle restait marginale par rapport au cœur de la Nouvelle-France, sont à reconsidérer ${ }^{23}$. Plusieurs de ces migrants sont demeurés sur les côtes de

22. Certains parleraient plutôt de déplacements pour cause professionnelle. Il n'en reste pas moins que des critères saisonniers, professionnels et extraterritoriaux s'ajoutent à la compréhension du phénomène. Pour une discussion du sujet, voir Abel Chatelain qui a établi une typologie des migrations dans «Problèmes de méthodes. Les migrations de la population», Revue économique, 14, 1 (1963): 1-17. Leslie Choquette a plus spécifiquement cerné la notion de migration saisonnière en fonction de la pêche en haute mer dans «Émigration et politique coloniale : les cas français et anglais », dans P. Joutard et T. Wien, dir., op. cit., 16-17, 179-180; Leslie Choquette, «L’Émigration vers la Nouvelle-France», dans Yves Landry, dir., Le Peuplement du Canada aux XVII et XVIII siècles (Caen, CRHQ-CNRS, 2004), 14.

23. Georges-Robert Bottin, "Sur les traces d'un flux migratoire maritime: Les Normands et la Gaspésie (XVIII et XIX ${ }^{\mathrm{e}}$ siècles)", dans Jean-Paul Hervieu et al., Les Normands et l'outre-mer..., op. cit., 251. 
la Nouvelle-France pendant un hiver, une année, quelquefois deux ans, mais il en est aussi un grand nombre qui y sont restés toute leur vie $\mathrm{e}^{24}$.

En effet, des recensements des côtes de la péninsule gaspésienne effectués en 1760 et 1761 confirment que 1153 habitants vivaient en permanence sur le territoire ${ }^{25}$. Or, on sait que ces gens étaient largement originaires de la Bretagne, de la Normandie et du Pays Basque et qu'un grand nombre d'entre eux s'y étaient installés depuis plus de vingt ou trente $a_{n} s^{26}$. Compte tenu de leur éloignement ou des circonstances particulières que représentaient à cette époque les périodes de tensions dans le golfe Saint-Laurent, ces hommes avaient-ils le sentiment de vivre en vase clos?

Rien n'est moins certain. Ils avaient notamment à subir les affres des guerres européennes qui venaient déstabiliser leurs opérations en Amérique $^{27}$ sans compter la concurrence que pouvaient leur offrir les capitaines pêcheurs originaires des autres régions de France qui menaient occasionnellement à de sérieux affrontements sur le terrain, notamment celle des Basques à Grande-Rivière ${ }^{28}$. Il existe d'autres preuves de la constance des liens entre l'Ancien et le Nouveau Monde qui tendent à montrer que les pêcheurs établis dans ce territoire ne semblent pas isolés. Le fait, par exemple, que les intéressés aux pêches passent indifféremment des marchés commerciaux ou autres documents du genre devant notaire à Québec ou en France. Citons comme exemple cette entente de Pierre Valleau consignée en France par laquelle il reconnait garder les chaloupes d'un morutier basque en pêche à Percé. Ajoutons à cela la vente de cordillat à Jean Boutillié, pêcheur et traiteur de Grande-Rivière, enregistrée à

24. D’ailleurs, les études n'ont pas manqué de souligner le fait qu'un petit nombre d'engagés demeuraient sur place durant l'hiver à Plaisance et Louisbourg, comme il en était en Gaspésie. Consulter à cet égard les ouvrages de P. Pope, de B. A. Balcom et de N. Landry, op. cit.

25. Selon le recensement de la Gaspésie en 1761 , la péninsule comptait $16,47 \%$ de la population de la Nouvelle-France alors qu'elle fait aujourd'hui 1,2\% de la population du Québec. On peut donc parler d'un peuplement d'importance pour la colonie. Nous concédons toutefois que, considéré du point de vue d'un métropolitain, le peuplement peut paraître marginal par rapport à la masse des pêcheurs qui ont traversé l'Atlantique. Régis Brun, "Recensement de Ristigouche 1760 et Livre du dénombrement des familles acadiennes réfugiées le long des côtes de l'Acadie 1761 ", Revue d'histoire de la Gaspésie, 8 (janvier-mars 1970): 30-36.

26. Yves Frenette, "Le peuplement francophone de la Gaspésie, 1690-1940: essai de synthèse», Gaspésie, 19, 3-4 (septembre-décembre 1991): 35-44. Mario Mimeault, «Le peuplement de la Gaspésie», Mémoires de la Société généalogique canadienne-française, 51, 4 (hiver 2000): 317-324.

27. Lire à cet effet nos contributions à propos de Pierre Revol, Jean Barré, Pierre-Léon Roussy, François Aubut, les pilotes du Saint-Laurent, François Lefebvre de Bellefeuille dans G. Deschênes et D. Vaugeois, op. cit.

28. M. Mimeault, Les Basques en Nouvelle-France, op. cit., $133 \mathrm{~s}$. Nous rappelons aussi en quelques pages les effets des grands épisodes de guerre qui ont bouleversé les côtes de la Gaspésie dans Mario Mimeault, La Gaspésie (Québec, PUL/IQRC, 2004), 70-76. 
Bayonne en 1752, ou les transactions, lettres et billets passés à GrandeRivière avec des gens de France que François Lefebvre de Bellefeuille dépose dans les minutes du notaire Jean-Claude Panet de Québec. Conséquemment, il est difficile de souscrire à l'idée que les travailleurs de la mer engagés dans les pêches de ce côté-ci de l'Atlantique Nord se considéraient coupés de leur pays d'origine, voire de leurs familles ou de leurs villages comme le croient certains auteurs ${ }^{29}$.

\section{LES LIENS DE PROVENANCE}

D’où venaient précisément ces personnes qui faisaient la navette d'une rive à l'autre de l'Atlantique? Pourquoi cette question? Parce que des chercheurs comme Jacques Mathieu ont établi que les liens de provenance expliquent souvent la présence des gens au cœur de la Nouvelle-France ${ }^{30}$. N'en serait-il pas de même pour les marins-pêcheurs venus des ports de Saint-Malo, de Granville ou d'ailleurs qui ont choisi de rester sur les rives du golfe Saint-Laurent? Il semble bien que oui.

Ces grands centres maritimes et leur hinterland, remarque Leslie Choquette, ont fourni comme bien d'autres l'essentiel des émigrants venus peupler le Canada. et on retrouve un grand nombre de ces ressortissants sur les bords du golfe Saint-Laurent. Cette émigration, fait-elle remarquer, était à la fois géographiquement restreinte et généralisée. Restreinte par l'étendue du bassin de recrutement des candidats et généralisée en ce qu'un grand nombre de villages y participaient. La zone d'influence de chacun de ces ports de mer s'étendait à des kilomètres autour des villes concernées (jusqu'à cinquante kilomètres pour Granville et Saint-Malo). Très peu des communes comprises dans ce rayon échappaient à l'attraction de l'économie atlantique. En Normandie et en Bretagne, les communautés agraires situées dans une telle mouvance et qui n’ont pas été touchées faisaient exception ${ }^{31}$.

29. Antoine Bernard: «... La Gaspésie n’est qu’un petit coin perdu, sur les confins de la province de Québec", Antoine Bernard, La Gaspésie au soleil (Montréal, Les Clercs de Saint-Viateur, 1925), 309. Jules Bélanger, «La Gaspésie dont l’isolement avait toujours freiné sinon bloqué l'évolution ... », dans Marc Desjardins, Yves Frenette, Jules Bélanger, Bernard Hétu, Histoire de la Gaspésie (Montréal, Boréal/IQRC, 1999), "Chapitre 15 : La Gaspésie nouvelle», 665.

30. Jacques Mathieu, Pauline Therrien-Fortier et Rénald Lessard, «Mobilité et sédentarité : stratégies familiales en Nouvelle-France», Recherches sociographiques, 28, 2-3 (1987): 211-227. Voir aussi Yves Landry, "L'émigration française au Canada avant: premiers résultats d'une microanalyse», dans Andrée Courtemanche et Martin Pâquet, dir., Prendre la route. L'expérience migratoire en Europe et en Amérique du Nord du XIVe au XXe siècle (Hull, Éditions Vents d'Ouest, 2001), 99, note 17.

31. L. Choquette, op. cit., 59-60, 76. 
La provenance rurale des pêcheurs posait-elle problème? Au premier abord, il pourrait surprendre que des gens de la campagne soient engagés pour des expéditions de pêche aux terres neuves, mais ce ne doit pas être le $\operatorname{cas}^{32}$. D'une part, les ports de mer ne pouvaient fournir à eux seuls tout le personnel recherché par l'ensemble des armateurs et, d'autre part, les villages de leur arrière-pays se trouvaient plus souvent qu'autrement aux prises avec un problème de surpopulation. Par ailleurs, et Thierry Sauzeau en a fait la démonstration pour les gens du Quartier de Marenne, en Saintonge, il se peut que les stratégies de groupe aient fait que l'on ait accepté le départ d'un fils en mer aux fins d'assurer un meilleur soutien à la famille ${ }^{33}$. Le fait en soi ne posait pas non plus de problème aux commissaires recruteurs, comme le souligne Charles de La Morandière, ces employeurs voyant chez ces ruraux une excellente relève après quelques saisons passées en mer $^{34}$.

Quoi qu'il en soit, il apparaît que la géographie de l'embauche chez les équipages de pêche épouse les mêmes caractéristiques que celles observées pour l'engagement des colons qui ont peuplé le cœur de la NouvelleFrance ${ }^{35}$. Qui plus est, il se peut que le bassin de ce personnel ait été encore plus restreint. Un coup de sonde réalisé sur l'origine des marins-pêcheurs de L'Union des Deux Frères, un navire de Granville parmi d'autres, révèle pour les années 1730, 1731 et 1732 un bassin de recrutement moyen de $17,4 \mathrm{~km}$ autour du port ${ }^{36}$. En analysant de près ces données, on remarque un certain regroupement géographique dans la composition de cet équipage, un phénomène qui se retrouve parmi tous les autres morutiers en partance pour la pêche lointaine. Des quatre-vingt-quatorze hommes embarqués sur le navire, quarante-trois venaient de Granville, onze de Bricqueville, cinq du Faubourg de Granville, cinq de Lingreville, quatre de Coudeville et ainsi de suite.

32. Pour s'en convaincre, il n'y a qu’à lire Alain Cabantous, «Des paysans pour la mer. La société des pêcheurs (vers 1600 - vers 1850)", dans Michel Mollat, dir., Histoire des pêcheries maritimes en France, op. cit., 183-242.

33. Thierry Sauzeau, «Les filières d'apprentissage des gens de mer aux XVIII ${ }^{\mathrm{e}}$-XIX ${ }^{\mathrm{e}}$ siècles», Techniques \& Culture [En ligne], 45, 2005 : 3, 9: http:// tc.revues.org/1393 (Consulté le 13 février 2014).

34. Charles de La Morandière, Histoire de la pêche française de la morue dans l'Amérique septentrionale, vol. I, op. cit., 97.

35. Jacques Mathieu, Pauline Therrien-Fortier et Rénald Lessard, «Mobilité et sédentarité... », op. cit., 213-214.

36. Un sondage sur trois autres navires, L'Aimable François de Granville (1743), Le Sauveur de SaintMalo (1720) et La Manon de Saint-Malo (1752) indique que le bassin de recrutement moyen devrait avoisiner les 21 à $22 \mathrm{~km}$ mais qu'il peut facilement s'étendre jusqu'à 33 kilomètres du port. Il arrive même que certains marins-pêcheurs viennent d'aussi loin que $100 \mathrm{~km}$ du lieu d'embarquement. Site Migration, op. cit., www.migrations.fr/naviredepartgranville1730.htm (Consulté le 17 octobre 2013). 
Il est évident, à la vue de cette concentration, que les liens de provenance ont joué dans le recrutement de ces marins-pêcheurs, comme il en fut dans celui des autres navires. Chaque commune a vu plusieurs de ses habitants s'embarquer pour l'Amérique chaque année et, ce, pendant toute la durée du siècle. Certains résultats du travail du généalogiste Pierre Provost appuient cette idée. En recensant les hommes qui venaient de la paroisse des Biards, en Basse-Normandie, il a relevé trente-sept noms de pêcheurs originaires de cette localité qui se sont enracinés en Nouvelle-France après être passés en Gaspésie entre 1720 et $1755^{37}$. Ce bourg comptant à cette époque 231 feux (environ 1000 habitants), on peut croire que tous ces gens se connaissaient bien et que les liens de provenance ont joué par la suite dans les relations tissées au Nouveau Monde ${ }^{38}$. Ce qui explique que François Breux, originaire des Biards, présente ses deux amis d'enfance Jean Thomassin et Jacques Mazière comme témoins à l'enregistrement de sa liberté au mariage et que Michel Charuet obtienne le même appui de Julien Sénéchal, un compagnon de travail qui a été élevé avec lui ${ }^{39}$.

Et même si les témoins viennent de villages voisins, les distances sont si courtes d'un patelin à l'autre qu'il est fatal que des marins-pêcheurs se reconnaissent une fois installés en Amérique. Nicolas Bry et Charles Gentil, de Saint-Pair, bourg situé à quarante-huit kilomètres des Biards, sont de ceux qui retrouvent François Aubut en Gaspésie, une vieille connaissance originaire de leur province. Les deux hommes racontent même avoir connu ses père et mère et avoir bu et mangé chez eux en sa compagnie $^{40}$. Ainsi devient-il évident, en raison de tels liens de voisinage, qu'il n'y avait pas moyen d'échapper à ses origines malgré les distances. C'est l'expérience que fit un novice du nom de Georges-René Pléville Le Pelley. Embarqué pour Gaspé à bord du Comte de Taurigny en 1739, il s'est enfui en fin de campagne en raison des sévices commis à son égard par le capitaine du navire. Le jeune homme, qui avait changé d'identité entretemps pour protéger ses arrières, se fera malencontreusement reconnaître par l'un de ses pairs à son retour sur un autre navire en 1741. Seules ses

37. Pierre Provost, "Ils sont venus des Biards», Site Internet Migrations, dans la rubrique «Pêcheurs et marins venus en Nouvelle-France»: www.migrations.fr/BIARDAIS/biardais.htm (Consulté le 19 septembre 2013). Il s'agit de la reprise d'un article publié dans Les Mémoires de la Société généalogique canadienne-française de Montréal, 4, 4 (hiver 1993): 275-279.

38. Saugrain Laîné, Nouveau dénombrement du Royaume par généralitez (sic), élections, paroisses et feux (Paris, Saugrain père, 1721), 65 : http: / books.google.ca (Consulté le 20 octobre 2013).

39. "Témoignages de liberté au mariage», dans Pierre-Georges Roy, Rapport de l'Archiviste de la Province de Québec, 1951-53 (Sans lieu, Imprimeur de la Reine, 1954), 69, 93.

40. Ibid., 12. 
hautes origines sociales et la bienveillance des officiers l'ayant pris en charge empêcheront des représailles ${ }^{41}$.

\section{LES LIENS DE FAMILLE ${ }^{42}$}

Tous ces éléments de démonstration n'ont ici qu'un but, établir qu'en s'installant sur les côtes du golfe Saint-Laurent ces gens ne pourraient avoir que difficilement souffert d'une coupure d'avec leurs origines. En d'autres mots, l'Atlantique est un monde de rencontres et de solidarités dont l'effet premier est d'atténuer l'impact de la séparation. On ne saurait se penser seuls dans un univers perdu ou abandonné des siens quand on est entouré de compatriotes. Il s'agit d'ailleurs là d'une vision de la migration qui nous amène à parler du phénomène de continuité dans l'accompagnement.

Certains modèles de comportement qui régissent les rapports personnels chez les migrants apparaissent nettement dans les rôles d'équipage des navires morutiers. Ces derniers révèlent au premier titre que la traversée d'un grand nombre de marins-pêcheurs français se faisait plus souvent qu'autrement à la faveur d'un accompagnement ${ }^{43}$. Accepter un engagement devant mener à des territoires de pêche situés de l'autre côté de l'Atlantique relevait d'une décision lourde de conséquences et qui se devait d'être mesurée. Les naufrages, les attaques ennemies, les décès par maladie ou

41. Georges-René Pléville Le Pelley, Mémoires d’un marin granvillais, 1726-1805 (Saint-Lô, Société d'Impressions d'Art Le Rachinel, 2002), (Les Cahiers Culturels de la Manche), 33. Site Migrations: www. migrations.fr/NAVIRES1755/lebrillant1755.htm (Consulté le 17 octobre 2013).

42. En 2010, une journée d'étude était consacrée à l'étude des mutations que l'espace atlantique imposait au lien familial tout en mettant à profit le regard croisé que pouvaient y porter la recherche française et nord-américaine : Journée coordonnée par Dominique Goncalvès et Zélie Navarro-Andraud, L'espace atlantique: rôles et impacts d'un monde sur ses acteurs: Le lien familial à l'épreuve de l'Atlantique, Université de Toulouse, le 12 février 2010: http:/ / calenda.revues.org/nouvelle15675.html (Consulté le 5 avril 2013). Jacques Perret et Thierry Sauzeau y ont apporté une contribution significative sur la condition familiale considérée dans le contexte transatlantique : Jacques Perret, «Les retours d’Amérique et la vie familiale au XVIII ${ }^{\mathrm{e}}$ siècle», op. cit.; Thierry Sauzeau, "La famille en Saintonge face aux métiers maritimes dans l'aire atlantique à la fin du XVIII ${ }^{\mathrm{e}}$ siècle», op. cit.

43. La notion de l'accompagnement dans la prise de décision des personnes en phase de passer à l'action se retrouve dans à peu près toutes les expériences migrantes. Voir quelques exemples dans: Mario Mimeault, L'Exode québécois, 1852-1925. Correspondance d'une famille dispersée en Amérique (Québec, Septentrion, 2013), 236-237. Nous traitions d'un accompagnement familial. Yves Landry relève dans la même optique le cas de 22 personnes recensées parmi les passagers du Saint-André (1659) parties ensemble pour aller coloniser Ville-Marie (Montréal). Yves Landry, «L'émigration française au Canada avant 1760 : premiers résultats d'une microanalyse» dans Andrée Courtemanche et Martin Pâquet, dir., Prendre la route, $85 \mathrm{~s}$. Lire aussi Fabien Raguy, L'émigration coloniale vers la Nouvelle-France aux XVII et XVII ${ }^{e}$ siècles dans le cadre de la reproduction sociale à Marans en Aunis, Mémoire de maîtrise (histoire), Université de La Rochelle, 96-100. La consultation des rôles d'armement reproduits sur le site Internet «Migrations » offre une multitude d'exemples de situations d'accompagnement professionnel qui indiquent une tendance significative. Voir Migrations, «Navires de nos ancêtres»: http://migrations.fr/naviresancetres.htm (Consulté le 26 mai 2014). 
accidents de travail, les disparitions en mer, toutes ces menaces qui guettaient les marins ne devaient pas avoir bonne presse dans les arrière-pays de Saint-Malo et de Granville, en fait de quelque port que ce soit.

Cet accompagnement prenait souvent la forme d'un mentorat.

Au sein de cette forme minimale de projet collectif [la traversée outre-Atlantique], mousses et novices étaient souvent parrainés par un matelot... Un véritable parrainage s'organisait... Les parrains exerceraient l'autorité tout en étendant leur protection paternelle sur les recrues les plus jeunes,

explique Thierry Sauzeau en parlant des équipages de la Saintonge, mais on peut aisément supposer que son observation vaut pour tous les équipages constitués en métropole ${ }^{44}$. Si, dans une telle aventure, la compagnie d'un ami ou d'un coparoissien avec qui partager les affres du voyage pesait dans la prise de décision, celle d'un proche parent devait être encore plus significative. Comme Yves Landry l'observe, le rôle d'entraînement de la famille dans la décision de partir et le choix de sa destination n'est plus à montrer $^{45}$. Son observation concernait le peuplement pionnier de la Nouvelle-France, mais nos sources indiquent qu'elle vaut tout autant pour la migration d'une main-d'œuvre «flottante», pour employer son vocabulaire, que pour ceux qui se sont installés à demeure sur les rives du golfe Saint-Laurent. Le cas des marins-pêcheurs qui ont traversé l'Atlantique avec un frère, un oncle, un cousin ou bien qui ont fait la navette entre leurs établissements de pêche en Amérique et le vieux continent en offre un bel exemple.

Les liens de famille remplissaient, en effet, une fonction de premier plan. La référence au même François Aubut, que nous venons tout juste de faire, illustre bien notre propos. Débarqué pour la première fois en Amérique à l'âge de seize ans, le jeune homme accompagnait un oncle homonyme de qui il apprit le métier de pêcheur avant de s'installer en permanence à Grande-Rivière ${ }^{46}$. L'histoire des frères Joseph et Olivier Nicolle offre un semblable exemple du phénomène de l'accompagnement

44. Thierry Sauzeau, «Les gens de mer de Saintonge, de l'Atlantique subi à l'Atlantique choisi (17601860)", Les Cahiers de Framespa [En ligne], 9 (2012): paragraphe 14: http:// framespa.revues.org/1223 (Consulté le 25 septembre 2013).

45. Y. Landry, op. cit., $84 \mathrm{~s}$.

46. Mario Mimeault, «François Aubut», dans G. Deschênes et D. Vaugeois, Vivre la Conquête, op. cit., vol. 2, 15-25. Pierre Provost, «La Saga de François Aubut pêcheur normand émigré en Nouvelle-France », Migrations: www.migrations.fr/AUBUT/familleaubut.htm; (Consulté le 21 octobre 2013). Dans l'étude des carrières des marins français ayant traversé en Amérique, il convient d'ajouter le recours au site de Charles Vianney Campeau, Navires venus en Nouvelle-France-gens de mer et passagers de 1700 à la Conquête: http: / / mwlandry.ca / genealog/ showsource.php?sourceID=S1473\&tree=05 (Consulté le 10 janvier 2014). 
parental chez les marins et pêcheurs français implantés sur les rives de la Nouvelle-France. Joseph, vingt ans, traversait à Gaspé pour la première fois en compagnie de son oncle Julien en 1749. L'année suivante, il reprenait la mer en compagnie de ses cousins Nicolas et Jean Coquet, puis, en 1755, il amenait avec lui son cousin Jean Beust (Lebœuf). Resté finalement en Amérique, Nicolas aura comme témoin à son mariage, parmi d'autres compatriotes, ce cousin Lebœuf ${ }^{47}$.

Il y a aussi ces cousins proches des frères Nicolle et de Jean Lebœuf, François et René Pelchat, encore des Biards, qui ont traversé l'Atlantique sur le même navire que François Aubut ${ }^{48}$. Plus encore, reproduisant en Nouvelle-France le schéma de proximité lié à leurs origines, tous ces proches parents, bousculés par la Guerre de la Conquête, se réinstalleront à quelques dizaines de kilomètres l'un de l'autre : les frères Coquet à Saint-François-dela-Rivière-du-Sud et à Saint-Thomas de Montmagny, Lebœuf à Saint-Vallier, les frères Pelchat aussi à Saint-Vallier et Aubut à Saint-Jean-Port-Joli" ${ }^{49}$.

Et on comprendra qu'il en est de même pour les autres réseaux des familles de pêcheurs qui se déploient en Amérique. Bref, il appert que les "loyautés familiales invisibles», pour emprunter au concept d'Yvan Boszormenyi-Nagy, tout en l'élargissant à l'environnement social du pêcheur, priment avant toute chose $^{50}$. Si l'accompagnement joue dans la traversée des gens de mer outre-Atlantique, les liens de famille se superposent souvent dans ce contexte à ceux de la provenance. Plus encore, ces attaches peuvent aussi se reproduire dans la relocalisation de ses membres en Amérique. Ainsi, s'est-il créé dans une projection spatiale joignant les deux rives de l'océan une toile éthérée mais bien réelle, une relation affective entre provinces d'origine d'un côté et lieux d'implantation de l'autre, entre familles demeurées en Europe et fils de famille installés au Nouveau Monde.

47. Jocelyne Nicol-Quillivic, «Les Rameaux d'Olivier - Mon ancêtre Olivier Nicolle», Site Migrations, www.migrations.fr/Les_Nicol.htm (Consulté le 30 octobre 2013). Voir aussi les rôles d'équipage des navires Le Prince d'Orange, (1749), du Jean-Alexandre (1754) et de La Favorite (1755).

48. "Témoignages de liberté au mariage»,op. cit., 95. Il n’y a pas que les témoignages de liberté au mariage qui en attestent. Nous retraçons les mêmes liens de famille ou de provenance à la lecture des annotations qui accompagnent les rôles d'équipage des navires morutiers de Saint-Malo et de Granville qui ont pris la mer dans les années 1720. Voir encore une fois Migrations, op. cit.

49. Éloi-Gérard Talbot, Généalogie des familles originaires des comtés de Montmagny, L'Islet, Bellechasse (Château-Richer, Frères Maristes, s.d.), vol. I, 63 ; vol. X, 32 ; vol. XII, 39, 43, 229, 23.

50. "Les loyautés familiales invisibles d’Yvan Boszormenyi-Nagy», dans Jean-Marie Lange, Une introduction à la psychologie relationnelle (Paris, L'Harmattan, 2005), 267-272. 


\section{LES COMMUNICATIONS ORALES}

Les liens de provenance, le réseautage familial, le mentorat professionnel, l'accompagnement sont tous des cadres structurels et comportementaux propres à assurer une forme de prolongement de ses attaches entre la province que l'on a quittée et les nouveaux espaces que l'on vient occuper. Il fut cependant une autre manière de donner vie à l'univers français qui transcendait l'océan Atlantique. En effet, au-delà de la compagnie de gens issus des mêmes milieux, on peut s'arrêter à la façon dont les pêcheurs ont alimenté cette relation affective avec leurs proches. Demandons-nous comment ceux d'entre eux qui s'étaient installés sur les rives du golfe Saint-Laurent se tenaient informés de leurs familles ou de leur province d'origine. Kenneth J. Banks écrivait que «to recognize the existence of a "French Atlantic" is, first of all, to understand the possibilities and limitations of seaborne communications ${ }^{51}$ ». Il faut comprendre ici que lorsque Banks parle de "communication», il entend autant les liens maritimes (navigation, commerce) que les mouvements de population et la transmission des messages. Nous avons traité des deux premiers éléments, les liens maritimes et les mouvements de population. Il reste la circulation des informations.

Dans cette optique, on comprend, en même temps que les navires et leurs équipages circulaient, que les renseignements transitaient eux aussi ${ }^{52}$. Ainsi, y avait-il pour les pêcheurs demeurés en Amérique deux manières de garder le contact avec leurs proches, soit de s'en informer en prenant de leurs nouvelles auprès des derniers compagnons arrivés d'Europe au printemps soit, encore mieux, en recevant des lettres de la famille ou en confiant des messages à des amis au moment de leur retour pour la France à l'automne. Comment cela se passait-il?

Aux fins de notre argumentation, revenons-en encore une fois aux traversées du navire L'Union des Deux Frères entre 1730 et 1732. Sur les quatrevingt-quatorze membres de l'équipage, quarante-huit personnes se déclaraient de Granville ou de son faubourg. Ce port de mer comptait un peu plus de 4000 habitants aux environs de l'année 1700, et certainement pas beaucoup plus, trente ans plus tard ${ }^{53}$. C'est peu et tout le monde devait

51. Kenneth J. Banks, Chasing Empire Across the Sea: Communications and the State in the French Atlantic, 1713-1763, 8. Et pour élaborer son hypothèse, Banks en réfère à James Carey, Communication as Culture, Essays on Media and Society (Boston, Hunwin Hyman, 1988), 14-21.

52. John Willis, dir., More Than Words. Readings in Transport, Communication and the History of Postal Communication (Ottawa, Canadian Museum of Civilization, 2007), 67.

53. L Choquette, op. cit., 276, note 34 . 
se connaitre. On a suffisamment parlé du va-et-vient transatlantique pour penser que les nouvelles se propageaient allègrement dans les réseaux de famille quand chacun des membres de l'équipage prenait ses quartiers à son retour. Les commérages, potins et autres échos de village créaient ni plus ni moins qu'un pont entre les communes françaises et les lieux de travail de leurs enfants outre-Atlantique. C'est là une autre manière de dire que ces rumeurs assuraient la continuité entre les deux littoraux.

Sans multiplier les exemples inutilement, citons le cas de la petite communauté de Viré, près de Granville. Forte de 271 feux en 1720, le village a vu dans la décennie nombre de ses concitoyens s'en aller pêcher sur les rives du golfe Saint-Laurent pour en revenir chaque automne ${ }^{54}$. Il est possible de croire que la communauté devait s'animer au retour de ses enfants. À l'opposée, de l'autre côté de l'Atlantique, un pêcheur comme Louis Le Normand, qui est resté à Grande-Rivière de 1750 à 1753, ne pouvait attendre qu'avec impatience le retour des pêcheurs originaires de son patelin, des gens comme Jean-Baptiste Fortin, Marin Durand, Nicolas Le Prieur ou Jean-Baptiste Poigné, pour recevoir des nouvelles de ses parents ${ }^{55}$.

Certaines situations demandaient cependant de se montrer un peu plus pro-actif dans la transmission des messages. François Aubut, le neveu qui avait traversé l'Atlantique avec son oncle homonyme, s'était installé à Grande-Rivière, mais il s'est retrouvé un jour dans une situation particulière. À la suite de quelques traversées annuelles, son oncle était revenu de France au printemps 1754 en compagnie d'un second neveu, en fait le frère du premier, un jeune enfant de douze ans. Malheureusement, l'oncle décéda au cours de la campagne et le problème qui se posa est le suivant: François Aubut, le neveu, devait-il laisser l'enfant s'en retourner seul à Granville et annoncer la nouvelle à la famille? Cet oncle possédait des biens importants à Grande-Rivière. Son petit frère pourrait-il en rendre compte adéquatement? Que faire? Écrire une lettre..., peut-être, mais la complexité de la situation demandait davantage. La solution? Comme tout résidant de la côte en avait la possibilité, il opta pour apporter luimême le message à la famille en s'embarquant pour la France à la fin de la campagne tout en profitant de l'occasion pour y ramener le jeune $\operatorname{garçon~}^{56}$.

54. Saugrain Laîné, op. cit.

55. Armement de L'Aimable Françoise de Granville allant à Gaspé, 1753, Site Migrations, op. cit. "Témoignages de liberté au mariage», op. cit., p. 43.

56. M. Mimeault, «François Aubut», op. cit. 


\section{UN RÉSEAU POSTAL EN PARALLÈLE}

La communication verbale et les contacts personnels ne sont toutefois pas les seuls moyens mis à la disposition des pêcheurs pour s'informer de leurs proches ou donner de leurs nouvelles. La lettre fait partie des autres possibilités. On a tendance à croire que ce milieu de travailleurs compte peu de gens instruits, d'autant que leur correspondance n'a pas été retrouvée. Ce n'est pas faux, mais il devait bien y avoir une proportion de personnes parmi les acteurs de la pêche qui savaient écrire. À défaut de documents probants, n'y aurait-il pas de traces d'un tel courrier? Faut-il rappeler le travail du chercheur louisianais Carl A. Brasseaux qui a démontré l'existence d'une correspondance entre familles dispersées par la Déportation de 1755, ce qu'il appelle des «lettres fantômes». Ces documents qui n'ont pas survécu, il en a trouvé la preuve par la mention répétée dans les échanges entretenus entre les diverses communautés acadiennes dispersées en France, au Nouveau-Brunswick et en NouvelleÉcosse ou bien en Louisiane et aux Antilles ${ }^{57}$.

Nous savons que sur les rives du Saint-Laurent, pendant la guerre de la Conquête, la circulation d'un courrier fantôme mettait les exploitants des postes de pêche au fait des derniers développements survenus sur un point ou l'autre de la côte gaspésienne. Si nous n'avons pu trouver de ces lettres originales, nous disposons tout de même de certains de leurs passages qui ont été enregistrés devant notaire. Grâce à eux, nous pouvons partager l'état d'esprit qui prévalait dans les circonstances. L'un des établissements les plus exposés étant celui de Mont-Louis, nous avons pu mesurer le désarroi qui animait l'un de ses exploitants, Michel Mahiet. S’adressant à son associé Joseph Cadet, Mahiet tient à lui faire part de «l'inquiétude et l'embarras ou il se trouve à ce moment et [qui] lui font réitérer la présente qui vous fera remis [sic] par Monsieur Aubert qui peut vous informer par luy meme des temps facheux qui regnent le long de la cote, les nouvelles de guerre et de tout ce qui sen paffe, ce qui me donne Beaucoup de peine ${ }^{58}$ ».

57. Carl A. Brasseaux, «Phantom Letters: Acadian Correspondence, 1766-1784», Acadiensis, XXIII, 2 (printemps 1994): 124-132.

58. BAnQ, Québec, greffe J.-C. Panet, le 4 février 1757, Déclaration de Joseph Cadet contre Michel Mahiet. Joseph Cadet est ce même marchand qui a regagné la terre de ses ancêtres en s'établissant dans le Poitou-Charente après la cession du Canada à la Couronne britannique en 1763. Une excellente biographie raconte la carrière de cet homme en Nouvelle-France, en omettant toutefois de parler de son implication dans le développement des pêches dans la colonie. Marc St-Hilaire, Alain Roy, Mickaël Augeron et Dominique Guillemet, dir., Les traces de la Nouvelle-France: au Québec et en Poitou-Charentes (Québec, Les Presses de l’Université Laval, 2008), 108-109. André Côté, Joseph-Michel Cadet (1719-1781) Négociant et munitionnaire du roi en Nouvelle-France (Québec/Paris, Septentrion/Éditions Christian, 1998), 400 p. Une courte étude, résultat du travail d'un chercheur français, présentait déjà le munitionnaire du 
Nous disposons de quelques autres éléments de correspondance qui tendent à montrer l'existence d'un courant épistolaire tout aussi bien développé entre les postes de pêche canadiens et les grands ports de France. Les relations des frères Georges, François et Pierre Lefebvre de Bellefeuille, détenteurs des droits sur la seigneurie de Pabos, à l'entrée de la baie des Chaleurs, nous permettent de l'affirmer. Tous les trois avaient acquis collectivement ce domaine en 1729, mais le départ de l'aîné de la famille, Georges, pour Saint-Malo, où celui-ci entreprenait une carrière d'officier dans la marine française, a donné lieu à un échange de lettres dont plusieurs ont heureusement survécu.

Par elles, non seulement avons-nous appris les développements qui ont trait à la propriété de la famille, mais aussi les modalités de correspondance qui prévalaient pour les deux frères. Le capitaine Jean Barré joue dans cette relation le rôle de l'intermédiaire à qui on confie le transport des lettres entre Saint-Malo et Pabos, lettres adressées d'ailleurs dans des termes assez généraux. Qu'on en juge par cette simple apostille: «À Monsieur Lefebvre de Bellefeuille - Seigneur de Pabos par Québec ou par la Baye des Chaleurs ${ }^{59}$ ». Ce que l'on remarque dans la correspondance de la famille, c'est que non seulement les informations échangées passent par un particulier, mais les messages empruntent souvent plusieurs voies. «J'ai bien reçu votre lettre datée du 30 juillet par le sieur Barré qui m’a très flatté de prendre de vos nouvelles, écrivait Georges Lefebvre. Je vous ai fait réponse par Blondel.» Par prudence, le correspondant précise même: «Écrivez moi je vous prie de Québec à Saint-Malo par duplicata dans plusieurs vaisseaux ${ }^{60}$.

Le procédé d'envoi postal est bien connu des historiens ${ }^{61}$. Il reste néanmoins que cette lettre témoigne du fait qu'il existait dans le monde des pêches atlantiques un échange d'informations de part et d'autre de l'océan

roi : Alfred Barbier, Un Munitionnaire Du Roi à la Nouvelle France, Joseph Cadet (Poitiers, Blais et Roy, 1900), 16 p. Mario Mimeault, «Les entreprises de pêche à la morue de Joseph Cadet 1751-1758 ", Revue d'histoire de l'Amérique française, 37, 4 (1984): 557-572. Voir aussi sur Cadet le site de L'inventaire culturel général du Poitou-Charente la rubrique «Sur les traces de la Nouvelle-France en Poitou-Charentes et au Québec»: http://inventaire.poitou-charentes.fr/nellefrance/nf/aller/ administrateurs.html (Consulté le 25 septembre 2013).

59. McGill University Libraries, Fonds de la famille Lefebvre de Bellefeuille, CH227. S205, Department of Rare Books and Special Collections, Lettre de Georges Lefebvre à son frère François, Du Grand Port (?), le 20 septembre 1747. Encore moins précise, il y a cette lettre du 21 mars 1764 envoyée par le même au même à l'adresse suivante: «à Monsieur Lefebvre de Bellefeuille, Seigneur de Pabos en Canada "

60. Ibid.

61. John Willis, "The Scale of Postal Communication in New France in context», 127-148. Bernard Allaire, "Le réseau de communications postales durant le régime français», dans J. Willis, More than Words, op. cit., 19-34. 
à des niveaux autres que ceux des gouvernements et des marchands. Nous sommes à même de constater en second lieu, par l'imprécision des adresses, qu'il se trouvait de multiples points de chute pour le courrier. De là, on peut en déduire qu'un réseau postal largement ramifié existait entre la côte de la Nouvelle-France, le cœur de la colonie et la mère patrie. Par ailleurs, en poursuivant notre questionnement, nous en sommes à nous demander si un échange de courrier personnel aurait existé chez les travailleurs de la base, les pêcheurs qui ont choisi de demeurer en Amérique.

\section{ÉCRIRE POUR SE PARLER}

Les simples pêcheurs avaient la possibilité d'utiliser le même canal que les marchands et les seigneurs pour échanger des nouvelles avec leur milieu d'origine en confiant leur correspondance à des capitaines. Ils pouvaient même profiter du passage d'un compagnon de travail en France pour lui confier leur courrier. On l'a vu en mentionnant le cas des capitaines Barré et Blondel qui transportaient les lettres de la famille Lefebvre de Bellefeuille. On est même en droit de se demander si ceux-ci n'auraient pas amené plusieurs missives à la fois.

Un cas précis nous permet de le croire. En mars 1757, un navire marchand de Louisbourg, Le Dauphin, quatre-vingts tonneaux, vingt-neuf hommes d'équipage, était capturé par un escadre britannique en patrouille dans le golfe Saint-Laurent. On trouvait 150 lettres à son bord, documents aujourd'hui déposés aux Archives nationales du Royaume-Uni, toutes adressées à des hommes de métier habitant Louisbourg. Sur les quatrevingt-quinze destinataires dont on connaît les occupations, trente sont des marchands, quinze des officiels et des militaires, vingt-six des gens de métier: des cabaretiers, charpentiers, gouvernantes, cordonniers, forgerons, officiers mariniers. Mais y avait-il des pêcheurs parmi eux? L'étude n'est pas claire à ce sujet, ce qui laisse place à une autre question : ces gens savaient-ils écrire, parce que le problème est que nous n'avons pas encore trouvé de ces missives échangées entre les membres de familles de pêcheurs répartis de part et d'autre de l'Atlantique ${ }^{62}$ ?

Le meilleur moyen pour le savoir est d'en référer à leurs contrats d'engagement envers un producteur, d'y voir si ces personnes savaient signer et dans quelle proportion. La signature peut en effet être considérée, selon le 
chercheur Michel Verret, comme un indicateur valable du degré d’alphabétisation ${ }^{63}$. Ainsi, l'étude des contrats paraphés par Jean Gatin dit Saint-Jean pour le poste de Grand-Étang entre 1715 et 1725 nous révèle que quatorze des quatre-vingt-quatre personnes ont signé leur entente avec l'entrepreneur. Cela nous donne un résultat de $16,6 \%$ de personnes qui savent écrire et signer. Ce taux d'alphabétisation se rapproche de celui avancé par Jacques Mathieu pour les gens de la campagne sous le Régime français, soit $20 \%{ }^{64}$. Nous avons aussi réalisé une semblable évaluation chez les pêcheurs engagés par les sieurs Michel Mahiet et Joseph Cadet, gestionnaires et seigneurs du poste de Mont-Louis (1752-1758). Ainsi, avons-nous pu observer que dix-neuf des soixante-trois signataires savent lire et écrire. Cela fait une moyenne de $33 \%$ de gens dans la communauté des travailleurs capables de rédiger une lettre pour eux-mêmes ou pour un $\mathrm{ami}^{65}$.

Mais allons encore un peu plus loin. Sachant qu'il existe un registre d'état civil couvrant les années 1751 à 1758 pour les bourgs de GrandeRivière et de Pabos, deux agglomérations largement peuplées par des gens de Bretagne et de Normandie, nous avons vérifié combien de personnes à qui on avait demandé de valider les actes de baptême, de mariage et de sépulture avaient apposé leur signature. Nous y avons trouvé vingt-quatre endossements sur une possibilité de quarante-deux. Si on enlève les missionnaires et la famille du seigneur, pour ne vérifier que la classe populaire, nous nous retrouvons avec dix-sept signatures, ce qui indique un taux d'alphabétisation de 40,4\% $\%^{66}$. Était-ce beaucoup? Michel Verret, qui s'est penché sur l'alphabétisation des gens de la ville de Québec entre 1750-

63. L'une des chercheures les plus investies dans l'étude de la signature, Béatrice Fraenkel, considère que cette dernière appartient avant toute chose à un moyen de valider l'écrit: Béatrice Fraenkel, $L a$ signature. Genèse d'un signe (Paris, Gallimard, 1992), 319 p. Il n'en demeure pas moins que le signataire doit posséder les bases de l'alphabet pour l'employer. Michel Verret pose les critères de la signature comme indicateur de l'alphabétisation: Michel Verret, "L'alphabétisation de la population de Québec», Revue d'histoire de l'Amérique française, 39, 1 (1985): 58-62. Voir aussi sa discussion du sujet dans «La signature comme critère d'alphabétisation », dans Michel Verret, L'Alphabétisation au Québec, 1660-1900 (Québec, Septentrion, 2002), 38-40.

64. Jacques Mathieu, La Nouvelle-France - Les Français en Amérique du Nord XVI ${ }^{e}$ XVIII siècle (Québec, PUL, 1991), 207-208.

65. Le lecteur pourra consulter sur les entreprises de Cadet, Mahiet et Gatin, de Mario Mimeault: "Les entreprises de pêche à la morue de Joseph Cadet 1751 à 1758 ", op. cit.; "Michel Mahiet et la pêche côtière au Mont-Louis, 1744 - 1759", La revue d'histoire de la Gaspésie/Gaspésie, XVII, 2 (avril - juin 1979): 4-19; "Regard historique sur les pêches V: Jean Gatin dit Saint-Jean, aubergiste et entrepreneur en pêche», La revue d'histoire de la Gaspésie XXVIII, 2 (juin 1990): 15-22. Les engagements auxquels nous référons pour établir nos statistiques sont tous cités dans ces articles.

66. BAC, MG9, B 8, volume 1, Registres de l'Acadie et de la Gaspésie, "Registre des baptêmes, mariages et sépultures des églises paroissiales de Sainte-Famille de Pabok [sic], de la Baie des Chaleurs, de la Grande-Rivière et autres lieux de la Gaspésie depuis Eshédiac jusqu’au Kamouraska » - 1751. 
1759, a établi que la proportion des gens instruits atteint $43,1 \%$, ce qu'il considère comme assez bas comme niveau ${ }^{67}$.

On peut aussi penser que c'est peu, mais c'est amplement suffisant pour alimenter un courant épistolaire. Rappelons que Carl A. Brasseaux a pu établir que de dix à vingt pour cent des Acadiens déportés savaient lire et écrire, ce qui a suffi pour alimenter un échange de courrier entre communautés ${ }^{68}$. Fort de ce constat, nous sommes parti à la quête de tels documents à la manière Brasseaux, espérant au moins relever la trace de lettres fantômes.

Ce sont les archives ecclésiastiques, en fait les témoignages de liberté au mariage, qui nous fournissent la preuve de leur existence. Et s'il nous est malheureusement impossible d'en connaître le contenu, nous savons tout de même qu'elles ont existé. Noël Le Torel, matelot de profession originaire de Menneville Le Bengar, près de Coutances, affirme sous serment le 17 octobre 1757 avoir apporté une lettre à André Laforge, pêcheur de profession établi à Grande-Rivière depuis 1753. Cette missive lui venait de son oncle homonyme. André Beaussier dit Tranchemontagne, soldat devenu pêcheur à Gaspé au cours de l'année 1758, nous semble privilégié à cet égard. Deux amis d'enfance, François Masson dit Francour et André Thierry dit Saint-André, originaires de Poitiers comme lui, disent avoir transmis à leur ami chacun une lettre de ses père et mère. Et les exemples ont vite fait de s'accumuler. Bien qu'originaire d'une paroisse voisine, Jean De Lentin affirme avoir apporté à François Fleury, de La Rondé, évêché de Coutances, des lettres de ses parents à quelques reprises alors que ce dernier travaillait sur la côte de Gaspé ${ }^{69}$.

Combien de pêcheurs ont reçu des lettres parmi ceux qui ont comme eux choisi de rester en Amérique? Nous ne le saurons sans doute jamais, mais la preuve de leur existence est là. Le lien épistolaire existait bel et bien. Le pêcheur qui recevait des nouvelles par écrit jouissait même d'un double avantage sur le plan de l'information. Le porteur de la lettre n'était pas qu'un anonyme fonctionnaire attitré à un service postal. C'était aussi un ami ou un coparoissien qui avait rencontré ses parents, qui avait échangé avec eux et qui avait vu de ses yeux leur condition et celle de son village. Le destinataire pouvait questionner le porteur de la missive et enrichir l'information qu'on lui apportait. C'est de la sorte que le message oral et la lettre s'ajoutaient conjointement à la panoplie des éléments qui

67. M. Verret, «L'alphabétisation de la population de Québec», loc. cit., 68.

68. C. A. Brasseaux, "Phantom Letters : Acadian Correspondence», loc. cit, 124-132.

69. "Témoignages de Liberté au mariage», op. cit., 27, 49, 126, 146. 
ont contribué à tisser le lien France-Amérique et à donner une densité à un univers atlantique français.

\section{CONCLUSION}

À la faveur des pages qui précèdent, nous apparaît une France à cheval entre deux rives. Nous y avons présenté, pour emprunter à Alain Cabantous, deux "mondes en mouvement caractérisés par l'interdépendance et l'interaction", ces deux mondes étant le golfe Saint-Laurent et le nord-ouest français $(\text { Bretagne et Normandie })^{70}$. Ce texte s'attachait à donner un aperçu du faisceau de connexions interpersonnelles développées entre les gens de mer dans le cadre de leurs relations intercontinentales.

Au départ, ces deux entités géographiques nous sont apparues étroitement réunies par un intense trafic transocéanique, mais surtout et avant toute chose par des liens commerciaux. Nous y avons vu différents modèles d'affaires intégrés dans une économie atlantique: des producteurs granvillais tirant partie d'une morue canadienne, des armateurs normands ou bretons exploitant des établissements gaspésiens, des marchands canadiens ayant recours à une main-d'œuvre française, des entrepreneurs métropolitains possédant une base opérationnelle outre-Atlantique et une tête de pont apte à faciliter les échanges avec la clientèle en France. Tous incarnent une facette ou l'autre des liens qui cimentent une économie halieutique intercontinentale.

La pêche éloignée fut aussi le moteur d'un important déplacement de population à la faveur duquel sont apparus différents parcours personnels. Des matelots ont traversé l'océan en allers-retours incessants. Des capitaines ont partagé leur carrière entre les côtes de la Nouvelle-France et celles de la métropole, alors que des pêcheurs ont choisi de s'établir à demeure sur les rives coloniales. Le résultat a été que des familles se sont retrouvées partagées entre les deux littoraux pour une saison, pour une année ou pour une vie, mais jamais ceux dont nous avons parlé n'ont perdu le contact. Ce qui nous fait écrire que l'Atlantique est un monde d'interactions et de solidarités.

Cette solidarité entre les gens établis sur le pourtour du golfe SaintLaurent et ceux demeurés dans leur province d'origine s'est maintenue suivant différents modes de rapprochement: le compagnonnage, le men-

70. Alain Cabantous, «Résistance de principe ou lucidité intellectuelle: les historiens français et l'histoire atlantique?», ACFAS, Congrès national des sociétés historiques et scientifiques. $133^{e}$ congrès, Québec, 2008. Nous tenons à souligner la confiance que le professeur Cabantous nous a manifestée en nous confiant le texte intégral de sa communication. 
torat, l'accompagnement, la présence de proches parents, des liens d'amitiés ou de provenance. Il a été établi de plus que les contacts personnels, les communications verbales et les échanges épistolaires ont contribué à garder vivants et même à nourrir les rapports avec les parents qu'on avait laissés derrière soi.

Au final, il s'est agencé dans l'univers atlantique une toile immatérielle composée d'intérêts et de liens interpersonnels qui ont réuni deux sousespaces régionaux aux attaches partagées, le golfe Saint-Laurent d'un côté et les côtes de la Bretagne et de la Normandie de l'autre. 